console typewriter. The third request transfers a card image from the card reader to the PP, and the fourth request feeds the status block to the $\mathrm{PP}$ as a check on the operation. (Once these requests have been placed in the control block, the PP will simply wait until the appropriate data and status information are returned from the $\mathrm{CP}$ The $\mathrm{PP}$ can then request reading of the second card.
In summary, the software system gives each PP a flexible system for initiating and scheduling data transfers between a variety of devices. By giving the PP the ability to initiate, queue, and monitor the transfer of data betwen any two devices, the system provides capabilities for the disposition of data which normally exceed the capacity of an independent minicomputer installation.

\title{
An experiment control computer system time shared by several laboratories*
}

\author{
JOHN KNIGHT $\dagger$, VICTOR COLBURN, DAVID OWENS, \\ LEE FREEMAN, DANIEL SYED, and WAYNE RASBAND \\ National Institute of Mental Health, Bethesda, Marylands 20014
}

\begin{abstract}
An experiment control computer system, operational for more than 2 years, is discussed. The system is multiprogammed, using a vendor supplied real-time operating system. Individual experiments employ multitasking-fast response functions are implemented in core resident tasks while interactive and other slow response functions are implemented in tasks that operate under time sharing. The areas of psychological research currently supported are concept formation studies, EEG evoked response studies, monitoring the autonomic nervous system, perception studies, and family interaction studies.
\end{abstract}

Since January 1971 the computer system discussed has enjoyed a prominent role in support of intramural research programs of the NIMH. The system, which is built around a $32 \mathrm{~K}$ word CPU and auxiliary disk storage, provides the fast response times typical of a dedicated single user system in a powerful multiprogrammed time-sharing environment. This configuration can be used concurrently to control and monitor a wide range of standard psychological experiments.

A general purpose computer with standard peripherals using vendor supplied software is at the heart of the system. The hardware is summarized in Table 1. The software includes a real-time operating system, language processors, and utilities.

Psychological protocols impose a range of differing requirements to be met by a supportive computer. The timeliness of required response varies from less than a millisecond for stimulus control and physiological data collection to several seconds for interactive parameter entry and subject instruction. Flexibility of input/output is required for the many types of equipment employed in psychological research. Finally, computational power extends from the capability to do simple computations to the more sophisticated complete

\footnotetext{
*This project is a joint effort by the Computer Systems Laboratory of the Division of Computer Research and Technology and the Technical Development Section of the National Institute of Mental Health in support of the intramura research program of the National Institutes of Health.

thequests for reprints should be sent to John Knight, Division of Computer Research and Technology, National Institutes of Health, Bethesda, Maryland 20014
}

experiment control. Heavily utilizing vendor supplied software, the system design accommodates these various requirements for multiple simultaneous users.

The system is multiprogrammed; this means more than one program can be run concurrently. If used as a purely time-sharing system, the computer could run perhaps 30 programs at once. The special demands of experiment control reduce the number of programs which can share the system by half. Time sharing is implemented by swapping the time-shared tasks from the desk for a time slice of execution on a round robin basis. The core-to-disk and disk-to-core swap necessary to interchange two tasks averages about $1 / 2 \mathrm{sec}$.

Varying response time requirements in the different phases of each experiment have led to extensive use of multitasking. The concept of multitasking is basic to the system design. Each experiment control program is reduced to a series of tasks; one task for each process necessary to control an experiment. For example, a task may control stimulus generation and data collection while another task prompts the operator for $S$ identification and the specification of runtime parameters. Some processes require fast response and are allocated to core resident tasks. Other processes are less time dependent and can be controlled by tasks operating under time sharing. Functions which depend on fast response are not executed under time sharing. These functions are isolated and given special treatment. By allowing the associated task to remain in core while awaiting an interrupt, it has been possible to insure that 
Table 1

System Hardware

\begin{tabular}{lrl}
\hline Computer & 1 & $\begin{array}{l}\text { 32K 16-bit words } \\
\text { Hardware memory protect } \\
\text { 72 levels of priority interrupts } \\
\text { Eight-block transfer channels }\end{array}$ \\
Secondary & 1 & Fixed head disk (1.6 M words) \\
Storage & 1 & Moving head disk (1.6 M words) \\
Backup Storage & 2 & Magnetic tape units (IBM compatible) \\
Peripherals & 1 & Card reader \\
& 1 & Line printer \\
& 1 & Plotter \\
& 12 & Teletypes \\
& 6 & Cathode ray tubes \\
Additional & 48 & Digital input lines \\
Data Paths & 48 & Digital output lines \\
& 32 & Analog input channels \\
& 8 & Analog output channels \\
& 4 & High-speed analog input \\
& 1 & External time of year clock \\
& 1 & External interval timer \\
\hline
\end{tabular}

the response occurs in less than a millisecond. A dedicated computer can respond slightly faster, but many paradigms in psychology are well satisfied with millisecond timing.

The above philosophy has been implemented on our $32 \mathrm{~K}$ word computer by allocating core as follows: $9 \mathrm{~K}$ time-sharing partition, $11 \mathrm{~K}$ system functions including the operating system, and $12 \mathrm{~K}$ fast response core resident code. Since most users are able to implement their fast response functions in less than $4 \mathrm{~K}$, three or more fast response experiment control programs can usually operate simultaneously. Additional programs such as compilations, slow response experiment control programs or data analyses can run under time sharing along with the time-shared portions of the fast response experiment control programs.

A simple reaction time experiment is described to illustrate system operation. The experiment is used to relate time perception to various $S$ states. Specific experimental protocol calls for eight testing periods consisting of nine trials per $\mathrm{S}$. There are three types of trials which are to be presented in random order. Essentially, a trial consists of a computer signal to the $S$ who responds by pressing a button appropriate to the signal as fast as he can. The experiment control task is triggered by the interrupt from the button and records both the elapsed time and the button number.

Here is one way to implement such an experiment in our time-shared multitasking environment. The experiment control program is composed of two tasks and uses two disk files. One task actually signals the $S$ and collects the data points. The other task interacts with the experiment operator to obtain the $\mathrm{S}$ identification. There is a history file which is used to record the collected data for later analysis and a communication disk file which serves as the link between the two tasks during an experiment run. The history file is also used to control the scheduling of Ss.
The flow of control begins with the operator communication task. First, a FORTRAN coded task in the time-shared partition prompts the operator for the current S's ID. Using the S's ID as an argument, the task fetches the S's record from the experiment's history file and determines which testing period is scheduled for that particular S. The FORTRAN code then generates the random order of trials and writes the appropriate instructions in the experiment's communication file. Finally, the time-shared task activates the stimulus control task and checkpoints itself. The fast response task is read into core and begins by reading its instructions from the communication file, then it signals the $\mathrm{S}$ for a trial. The fast response task remains core resident as long as any interrupt from the $S$ is pending or until a time-out interrupt is triggered. After each trial, the core resident task writes the results in the communication file, activates the time-shared operator communication task, and terminates itself. The time-shared task reads the communication file and takes any corrective action necessary. It updates the S's record and then either informs the operator that the run is complete or proceeds with another trial.

In this implementation of the experiment, the intertrial period is assumed to be long enough $(10 \mathrm{sec})$ to safely allow for activation of the time-shared task. If the intertrial period is required to be shorter, the core resident task could run multiple trials with a single activation.

Once the experiment is complete, the data must be analyzed. Some researchers do their analysis locally, using the time-sharing partition. Others prefer to format the data on magnetic tape and take it to our central facility where generalized statistical programs and other powerful resources are available. To facilitate this last step, and also to provide complete experiment control, telephone links to the IBM 370 and PDP-10 computers of the NIH central computer facility are being developed.

The addition of the communications link will make the full computational power of the large computers easily available to the researcher. The goal of the communication design is to make experiment control extending from data collection through complete data analysis a smooth and timely process. This goal will be achieved by having the local computer and the users' terminal act as a "smart" terminal to the central facility. The "smart" terminal will be able to collect data, handle procedural problems and transmit results to the large computer for analysis and storage.

The reaction time experiment is only an example of typical computer assistance. In fact, a wide range of protocols and researchers receive support. A complete list of the programs supported by the computer appears in Table 2. The EEG evoked response experiments, for instance, require accurately timed intervals for stimulus generation and collect 1000 data points per second. The data editing functions of the system require the use of 
CRT displays and access to large magnetic tale files. The perception studies use an elaborate digital input/output device. A progam which has been developed to study family interaction employs five cooperating tasks. The concept formation program involves long sessions of computer assisted instruction and interactive publem solving using a special purpose terminal. The system services all of these requirements in various combinations simultaneously and its full potential is not being exerted.

The most frequent approach to laboratory automation has been to use a small dedicated computer for the collection of data, a trained assistant to handle procedural details of the experiment and a large data processing facility machine for subsequent analysis of the data. In such an approach, the terminology "small dedicated computer" may refer to a machine smaller that that used at NIMH, for instance, a 12-bit machine, but in general the actual machine class may be the same and the definition simply implies a configuration limited both in core size and peripheral equipment. Prior to the current installation, this approach was used by NIMH researchers, two investigators using a small LINC computer and three scientists sequentially sharing an IBM 1620 machine. Where a number of users are involved, it is felt that the system described in this paper provides significantly enhanced support over that provided by a number of small dedicated machines.

By pooling their resources, several users can afford substantial main memory and versatile secondary memory including magnetic tapes, fixed head, and floating head disks. The size of core makes the use of various language processors not only possible but practical. An extended FORTRAN is the most heavily used language on the system. The trace facility in FORTRAN and vendor supplied interactive debugging package greatly facilitate program development. The convenience of a card reader and a line printer offer a welcome contrast to paper tape and Teletype based systems. Using the overlay facility, experimenters can write programs of practically unlimited size. The speed and ease with which large programs can be developed, together with the greater computational power of the larger computer, make possible more efficient utilization of a researcher's time.

The most significant benefit gained in the transition from the dedicated computer to the shared computer approach is the possiblity of complete experiment control. As illustrated in the reaction time experiment, the computer under time sharing can assume many functions formerly done by a trained operator. For example, computer assisted instruction is used in connection with the data collection resulting in more uniform subject training. A modest amount of analysis can also be accomplished on the system during an experiment. In the absence of a large scale computer system, all of the analysis could be done on the local computer. Coordinated operation with a large data
Table 2

Applications Summary

\begin{tabular}{|c|c|}
\hline Area & Short Description \\
\hline & Computer-controlled stimulus causes changes in \\
\hline $\begin{array}{l}\text { Response } \\
\text { (Buchsbaum) }\end{array}$ & $\begin{array}{l}\text { S's EEG. Correlations have been found between } \\
\text { these changes and various other characteristics } \\
\text { of the } S \text {. }\end{array}$ \\
\hline $\begin{array}{l}\text { Perception } \\
\text { Studies } \\
\text { (Carlson) }\end{array}$ & $\begin{array}{l}\text { Ss take action in response to a controlled } \\
\text { stimulus. S's actions and physiological state } \\
\text { reflect S's perception of the stimulus. }\end{array}$ \\
\hline $\begin{array}{l}\text { Concept Form- } \\
\text { ation Studies } \\
\text { (Jerome) }\end{array}$ & $\begin{array}{l}\text { Ss required to interactively solve problems } \\
\text { which involve the formation of concepts. Each } \\
\text { guess made by } S \text { is saved for later study. }\end{array}$ \\
\hline $\begin{array}{l}\text { Family } \\
\text { Interaction } \\
\text { Studies } \\
\text { (Reiss) }\end{array}$ & $\begin{array}{l}\text { Families arc given problems to solve inter- } \\
\text { actively via one Teletype per member. Com- } \\
\text { munication between members is controlled by } \\
\text { the computer. Effects of family interaction in } \\
\text { problem-solving behavior are studied. }\end{array}$ \\
\hline $\begin{array}{l}\text { Monitoring of } \\
\text { Autonomic } \\
\text { NS (Zahn) }\end{array}$ & $\begin{array}{l}\text { Large amounts of multichannel data collected } \\
\text { off-line are edited using a CRT for later } \\
\text { computer processing. }\end{array}$ \\
\hline
\end{tabular}

Note-Researchers are in parentheses.

processing system is only possible because of the multiprogramming design and flexible input/output configuration.

Contrasting the speed and response time between shared and dedicated computer systems poses considerable difficulty, as the advantage fluctuates substantially with the size of the problem to be executed. Quite naturally, the absence of program overhead permits the dedicated machine to excel on those problems that are sufficiently small to be handled as core resident problems. As program size, including required data manipulation, exceed this limit, performance deteriorates drastically, since the dedicated machine must then rely on a limited selection of slow peripheral devices.

Relative to a small single user dedicated computer, the added complexity of a multiprogrammed system leads to a small reduction in reliability, since there are more functions to be accomplished. Importantly though, little of the complexity of the system is passed on to the user. Although the scientist must learn a job cortrol language, it is simple and easily learned in a couple of hours. Experience with the system at NIMH shows that scientists have little trouble learning to program or run the system by themselves. Notwithstanding this, it is less complicated to develop simple reliable programs on a dedicated system. It is not until complex tasks are attempted that the balance shifts in favor of the shared computer.

The questions of cost and manpower, are, of course, crucial to the evaluation of any system. Because of funding problems, the NIMH system was originally procured under a combination purchase and lease with option to purchase arrangement. On a total purchase basis, the system represented an $\$ 240 \mathrm{~K}$ investment. 
Since the purchase of this system in July, 1970, advances in computer technology have substantially reduced the cost of both computer and peripheral devices to the point that the current duplication cost should be around $\$ 25 \mathrm{~K}$. By the same token, a small dedicated system of restricted capability may be obtained for less than $\$ 25 \mathrm{~K}$ today. The greatest disparity, however, arises in consideration of the manpower resources required. A single competent engineer/operator can certainly satisfy the requirements of a dedicated system user if electronic interfacing requirements are limited. Although hardware effort may be assumed to be equivalent, the multiprogrammed time-shared system presents quite a different picture because of the sophisticated software involved. To date, the subject system has incurred approximately 8 man years of system programming. Three years ago, sophisticated systems software for small or medium size computers was the exception rather than the rule. Today most manufacturers have fully developed monitors and executives that have undergone considerable use and improvement. These systems must be adapted to the specific problem, however, and it is likely that a system similar to that in NIMH could be developed today with less than 6 man years of system software effort. Development of such a system should not be attempted without this resource.

A series of small computers attached to a very large time-sharing system is another approach to laboratory automation. Its major drawback is that a very large computer system must be available. The approach discussed can stand alone without the support of a large facility. It has the added advantage that should a large computer facility become available, the shared computer can make use of it.

There are several alternatives available for the automation of a psychology laboratory. One approach is for a group of researchers to pool their resources and share a time-shared computer which is capable of servicing their real-time needs. This approach has been implemented at NIMH and is workable. A major benefit which is unique to the medium size time-shared computer approach is that much of the problem can be solved on a single computer. If more than one computer is required, this approach provides an easy to use interface to the other computers.

\title{
Design and organization in a computerized psychology laboratory*
}

\author{
JERRY C. FORSHEE \\ Indiana University, Bloomington, Indiana 47401
}

\begin{abstract}
This paper presents six principles of laboratory organization and design. These six principles are an outgrowth of the past 7 years' experience in the Mathematical Models Laboratory of the Psychology Department at Indiana University. Each of the six principles is formally stated and then followed by explanation and examples.
\end{abstract}

\section{INTRODUCTION}

The several principles of organization and design for a computerized psychological research laboratory, presented here, are the result of several years of experience. The Mathematical Models Laboratory of the Psychology Department at Indiana University has been in operation since 1966 . The laboratory computing facility is centered around an IBM 1800 Process Controller, the configuration has grown to include the CPU with $32 \mathrm{~K}$ of core, 12 levels of priority interrupt and multiple process $\mathrm{I} / \mathrm{O}$ points, several standard peripherals, a card read/punch, a line printer, a dual disk

\footnotetext{
*The research facility decribed herein and from which these ideas evolve is supported by Public Health Service Grant PHS RO1 MH 16817.

tRequests for reprints should be sent to Jerry C. Forshee, Department of Psychology. Indiana University, Bloomington, Indiana $\mathbf{4 7 4 0 1 .}$
}

drive, and several typewriters. Other peripherals on the system include two computer terminal facilities consisting of a CRT terminal, a character printer, and an incremental digital tape drive. This computing facility is used to support four time-shared subject running rooms. The research community using this facility may include, in a given semester, as many as three or four full time faculty and from five to ten graduate students.

\section{The Principle of Accessibility}

\section{THE PRINCIPLES}

The principle of accessibility states that information about the current state of the computing system must be immediately available to the user.

This principle demands a facility such that if the user needs to know the values of Core Locations 1-50 he needs only to enter on a terminal the command DUMP 